\title{
EL CONTADOR PÚBLICO FRENTE A LA CUARTA REVOLUCIÓN INDUSTRIAL
}

\author{
THE PUBLIC ACCOUNTANT IN THE FACE OF THE FOURTH INDUSTRIAL \\ REVOLUTION
}

\section{RESUMEN}

Objetivo: Evidenciar si los Contadores Públicos en el Perú están capacitados para enfrentar la cuarta revolución industrial y analizar las competencias profesionales de la contaduría para prestar servicios en los entes económicos digitalizados. Método: Investigación cualitativa, documental no experimental, por cuanto no se ha manipulado las variables; sino que se ha observado el fenómeno objeto de estudio a través del análisis, la crítica y la comparación de diversas fuentes de información, considerando autenticidad, credibilidad, representación y significado. Resultados: La revolución industrial 4.0 no sustituye a los Contadores Públicos, pero sí exige nuevas competencias. Los Contadores Públicos no están preparados aún para afrontar los retos de la cuarta revolución industrial. Conclusiones: Los Contadores Públicos en el Perú deben estar en una constante capacitación holística, actualizados a los cambios tecnológicos, con formación interdisciplinaria, con conocimiento normativo y desarrollo de habilidades blandas.

Palabras clave: Competencia digital; Contador Público; Contaduria; Revolución 4.0
JuLio Gómez Méndez

Universidad Nacional de San Cristóbal de Huamanga Ayacucho, Perú

ORCID:http//orcid.org/0000-0002-0379-0349 Correo electrónico: julio.gomez@unsch.edu.pe

Nerio Janampa Acuña Universidad César Vallejo Ayacucho, Perú ORCID:https://orcid.org/0000-0003-0252-2649 Email: njanampa_5@hotmail.com

[Recibido: 09/06/2020 Aceptado: 21/07/2020 Publicado: 31/08/2020]

\begin{abstract}
Objective: Evidence of whether Public Accountants in Peru are trained to confront the fourth industrial revolution and analyze the professional competencies of accounting to provide services in digitized economic entities. Method: The research is qualitative, non-experimental documentary, so far as the variables have not been manipulated, but the phenomenon under study has been observed through the analysis, criticism and comparison from several sources of information, considering authenticity, credibility, representation and meaning. Results: The Industrial revolution 4.0 does not replace the public accountants but it does require new competences. Public Accountants are still not prepared to deal the challenges of the fourth industrial revolution. Conclusions: Public Accountants in Peru must be in constant holistic training, updated to the technological changes, with interdisciplinary training, with normative knowledge and soft skills development.
\end{abstract}

Keywords: Digital Competition; Public Accountant; Accounting; Revolution 4.0.

(c) Los autores. Este artículo es publicado por la Revista Quipukamayoc, Universidad Nacional Mayor de San Marcos. Este es un artículo de acceso abierto, distribuido bajo los términos de la Licencia Creative Commons Atribución-NoComercial-Compartirlgual 4.0 Internacional.(http://creativecommons.org/licenses/by-nc-sa/4.0/), que permite el uso no comercial, distribución y reproducción en cualquier medio, siempre que la obra original sea debidamente citadas. 


\section{INTRODUCCIÓN}

Entender la evolución de la labor del Contador Público en el mundo remite a la más antigua práctica de registros de actividades de trueque en tablillas de barro en Sumeria (6000 a. C.); y sin duda, a 1458 como nacimiento de la partida doble con Benedetto Cotruglli, inmortalizado en el Tractus XI-Particularis de computis et scripturis por el franciscano Luca Pacioli en 1509. Ese rol registral de transacciones asignado se transformó sustancialmente con la industrialización, sumándose el desarrollo de la auditoría para dar fe y velar por los recursos públicos en apoyo al Estado. En consonancia con el avance de la humanidad, las empresas y los modos de negocio fueron modernizándose, fue imperativo, por tanto, la transformación del rol del Contador Público. Es en ese contexto, que el marco normativo globalizado nace en 1973 con las Normas Internacionales de Contabilidad (NIC), como lenguaje financiero común, marcando un hito, tal vez el más importante en la historia para el Contador Público: el fortalecimiento de la fe pública (Quijano, 2020).

Al mismo tiempo en que la profesión contable experimentaba su globalización, aparecía en la aldea global la llamada revolución 4.0 trayendo consigo la inteligencia artificial como una de las tecnologías clave en la profunda transformación de la economía, la sociedad y el mercado laboral, que incide imperativamente. Los incesantes y exponenciales avances en las Tecnologías de la Información y la Comunicación (TIC) dieron lugar a la aplicación de la inteligencia artificial capaz de generar resultados con la velocidad necesaria para comunicar en el mercado (Blanco, Castro, Gayoso y Santana, 2019).

La transformación dirigida a la industria 4.0 se refiere a las habilidades para unir las tecnologías, alcanzar la mejora de diversas actividades y ser más productivo, pues a través de la innovación se logra el crecimiento. Es una transformación necesaria que toda organización debe asumir para agilizar sus operaciones y ser competitiva en el mercado, donde las demandas son cada vez mayores, pues las empresas están llamadas a mejorar sus procesos (Deloitte Insights, 2018).

La revolución industrial 4.0 transforma el modo de producción y el comercio, incluso las relaciones de los colaboradores con las empresas. Hay discrepancia ente los que asumen que la cantidad de colaboradores se reducirán en contraposición con los que mencionan que serán más. La discrepancia radica en que la educación es una manera de aprendizaje que debe prepararlos para enfrentar esta realidad. Dentro de las posturas optimistas sobre la cantidad de oferta de empleo y número de trabajadores, Amésquita (2018) señala que "lo cierto es que la educación debe enrutarse por los conocimientos y competencias más de planificación y diseño que enfrenten la rápida obsolescencia del conocimiento y la tecnología y hagan viables los nuevos negocios y empresas" (p. 196).

Existen dos tendencias sustantivas respecto de las empresas y la asimilación de las tecnologías 4.0. Una de ellas toma en cuenta el cambio de la manufactura en las áreas que desarrollan los productos y la ingeniería relacionada con la data que se emite desde el entorno digital de las máquinas para la toma de decisiones en tiempo real. Por otro lado, como lo afirman Basco, Beliz, Coatz y Garnero (2018) "en todos los sectores de la industria, la integración con proveedores, clientes y otros socios de la cadena de valor avanza más lentamente que la integración entre las áreas internas de la empresa" (p. 30).

La cuarta revolución industrial pretende abarcar campos más completos como los aspectos relevantes para el desarrollo de una empresa que realice sus actividades productivas apoyándose en elementos de las TIC para realizar con mayor precisión todas sus acciones, dentro de ellas encontramos la nanotecnología y la tecnología cuántica. Lo que busca esta revolución es unir las ventajas de todos los tipos de tecnologías para que sean aprovechadas por las organizaciones para hacerlas más rentables y funcionales que las empresas predecesoras. Esta revolución se está propagando por el mundo a mayor velocidad y amplitud; sin embargo, las anteriores revoluciones aún están en desarrollo, como la segunda, que revela su ausencia en un $17 \%$ de habitantes del planeta que no acceden a la electricidad, ya que como señala Schwab (2016) el no alcanzar la tercera revolución tiene a "más de la mitad de la población mundial, 4.000 millones de personas, la mayoría en el mundo en desarrollo, sin acceso a internet" (p. 13).

La cuarta revolución industrial trae consigo tecnologías nuevas que permiten fabricar productos y prestar servicios en formas y en lugares completamente nuevos, siendo la conectividad la característica principal que une a estas tecnologías y las hace auténticamente disruptivas. Para Blanco, Castro, Gayoso, y Santana (2019) existe una interacción conectiva y masiva entre personas y objetos a través de internet que genera "grandes conjuntos de datos relativos a su estado y a las transacciones e intercambios efectuados con otras personas y objetos; datos que, a su vez, también residen en internet y son susceptibles de ser empleados para construir nuevos productos y servicios" (p. 6).

La cuarta revolución industrial busca cambiar las interacciones de producción, los procesos de comercio e, incluso, las formas de existencia de las poblaciones que son consideradas las fuerzas de trabajo. Es un cambio que busca la transformación de todos los elementos que interactúan dentro de un mismo contexto, pues las indagaciones concluyen 
que las nuevas realidades demandan nuevas habilidades en las personas y es medular que posean conocimientos interdisciplinarios para ser más eficientes en sus actividades. Las habilidades deben desarrollarse como parte de la convivencia para que sean transferidos a través de las culturas y costumbres de un grupo de personas que buscan insertarse en el mundo laboral de existencia global. Por consiguiente, se considera que la educación es un mecanismo que debe proponer aprendizajes que les sirva para interactuar con el contexto que demanda ciertas habilidades para ser eficiente (Echeverría y Martínez, 2018).

Para los contadores públicos, el arribo de la cuarta revolución industrial plantea un dilema, común para las profesiones: Cuarta Revolución Industrial o Revolución 4. 0, ¿amenaza u oportunidad? La contabilidad como ciencia no ha escapado a los cambios de la globalización y la internalización digital, como profesión ha tenido que expandir sus horizontes y abrirse a la internalización de los mercados financieros, a los adelantos de las TIC y la Big Data que le exige mantenerse acorde a las exigencias de la organización empresarial y los usuarios de la información (Changmarín, 2019).

Con el auge de la implementación de los Enterprise Resource Planning (ERP), a finales del siglo XX e inicios del XXI, los Contadores Públicos tienen el imperativo de desarrollar nuevas habilidades y competencias para gestionar los sistemas de información. Para llenar los vacíos intelectuales, desde la academia y los colegios profesionales, se propuso la adopción de los avances tecnológicos globales. En la mayoría de los países de nuestra aldea global se han emitido normas legales con el propósito de la regulación de la trasmisión de datos y mensajes digitales, así como los negocios electrónicos, sin mucho éxito. Aun así, el contador público debe ser amplio conocedor de la data digital y lograr la evaluación válida de la sobredosis de información digital (Gómez, 2019).

Los tiempos actuales exigen nuevas formas de hacer negocio y de realizar transacciones comerciales en un entorno digital, así como el reemplazo de algunas labores en el quehacer de contaduría y la aparición de nuevos, por ello, el contador público tiene que reconvertirse, para ello debe desarrollar nuevas competencias y cualidades para realizar gestiones de negocios: los profesionales de la contaduría conscientes de estos nuevos cambios y retos seguirán siendo considerados actores necesarios. Frente a este panorama de cambios exponenciales y vertiginosos, quienes forman a los futuros profesionales vinculados a la producción y los servicios deben considerar ajustes prioritarios. Para Mayor, Pacheco, Patiño y Ramos (2018) la carrera de contabilidad tiene el desafío de fortalecer destrezas relacionadas con el Big Data, factor esencial para alcanzar nuevas propuestas de innovación en el procesamiento de la data. Por eso los programas universitarios de contaduría pública deben incluir en su malla curricular cursos relacionados con el Big Data y graduar profesionales contables con habilidades en esta tecnología y aportar a la innovación y progresos a la productividad y competitividad.

Los estudios y tendencias, a nivel general, revelan que las áreas de primera significancia en el quehacer contable seguirán siendo la auditoría y el reporte corporativo. Aun así, urgen dinámicos y pertinentes cambios en la competencia profesional del contador, debido a la nueva metamorfosis tecnológica: interconectividad en la nube, data interactiva, tecnología blockchain, el IoT. Para que la megadata que se obtiene con facilidad sea útil como información de valor, debe ser objeto de análisis integrado sistemático. Con agudeza, afirma Gómez (2019) que "la verificación del cumplimiento y el aseguramiento de la información requerirá conocimientos en sistemas expertos y capacidad para evaluar transacciones sin rastro físico, con base en operaciones y flujos electrónicos en tiempo real" (p. 2). Para su concreción es vital considerar la aplicación de un software que analice los datos, experticia de síntesis e interpretación y, fundamentalmente, un pensamiento globalizante e integrador. La interdisciplinariedad es imprescindible, sobre todo con profesionales de ingeniería y análisis de data.

Respecto a los contadores públicos y la inteligencia artificial, Ceballos (2018) sostiene que las funciones de los nuevos profesionales de la contabilidad deben ser direccionadas hacia el servicio con valor agregado. Sugiere el autor que los profesionales del área deben desarrollar habilidades que sean útiles para afrontar los retos de la industria 4.0 y ser competitivos en el mercado laboral. Para conseguirlo, se debe considerar siete puntos básicos. Lo primero es verificar si en el giro de la propia responsabilidad, el personal está preparado para asumir los retos. La recomendación número dos es tener una preparación multiáreas de modo que se desarrolle un mayor valor agregado intelectual. Un tercer consejo es potenciar las habilidades sociales blandas comunicativas. La cuarta recomendación es la consideración de alianzas estratégicas con otros profesionales de contabilidad y afines. El quinto punto por considerar es aplicar la neurociencia y las finanzas en la toma de decisiones. El sexto tip es vender la calidad profesional, los propios servicios y finalmente, la última consideración es valorar la competencia profesional actualizada lograda con esfuerzo de inversión personal y que las empresas o usuarios deben de reconocer al contratar los servicios profesionales del contador.

Actualmente, la evaluacion tecnológica permite un proceso de interacción de informacion digital entre actores de la economía y las organizaciones productivas y de servicio que ha revolucionado al mundo globalizado sin 
posibilidad alguna de normativas. Aquí se está en ventaja o desventaja. Ante esta situacion los profesionales de contabilidad deben de sumergirse en esta nueva forma de actuación digital demostrando competencia; así como señala Grisanti (2017) hay que tener en cuenta "que esta impronta tecnológica también puede facilitar y permitir a los contadores públicos realizar su trabajo de una manera mucho más eficiente y completa” (p. 116).

Es prioridad y vital que el experto contable y auditor se adapte y reconvierta en el nuevo contexto y coyuntura digital. Por consiguiente, los contadores públicos, ante este nuevo panorama, en primer lugar, tienen que romper sus añejos esquemas mentales para luego dar inicio al proceso inexcusable de reconversión en sus competencias y habilidades profesionales y seguir vigentes en este mundo de la revolución 4.0 (Grisanti, 2017).

La contaduría digital emergió y despega violentamente creando prácticas disruptivas en el ejercicio profesional. Los contadores tradicionales quedan fuera, desplazados con la contaduría digital. Se ha desatado una ola alarmista, ya los gremios que agrupan a los contadores están iniciando conscientemente espacios de capacitación continua e innovación para lograr competencia digital. Para Mantilla (2019), quien analiza la situación en Colombia, estos cambios disruptivos se entienden de manera equivocada o distorsionada "porque se siguen confundiendo las cosas pensando que: auditoría independiente es lo mismo que revisoría fiscal, normas es lo mismo que estándares, lo fiscal/tributario es igual que financiero, y que, el contador público es lo mismo que contador profesional” (p. 3).

Peña (2018) afirma que esta nueva vorágine tecnológica ha hecho girar a la economía mundial, y que "el enfoque principal de la era digital es el uso de la tecnología en diversos contextos y la eliminación progresiva de labores manuales o hechas hasta hoy exclusivamente por humanos" (p. 8). Reflejo de ello, se vislumbra un permanente y acelerado cambio en los negocios a todo nivel, involucrando a nivel tributario y fiscal, a las organizaciones empresariales y los estados, respectivamente; se ven sometidos a un cambio adaptativo.

El mundo ha cambiado, no es el mismo, esa transformación se debe a la dinámica tecnológica; en apariencia se presenta con mucha normalidad y a veces se cree ser parte de ello solo por interactuar en redes sociales. La realidad objetiva, sin embargo, es otra; las TIC han modificado usos y costumbres, gustos y preferencias. En el ámbito de la economía, las finanzas y los negocios que mueven a este mundo, "el Contador Público tendrá que adquirir los conocimientos necesarios para que domine muy bien la Red, ya que próximamente será él el encargado de publicar la información en ella" (Florez, 2012, p. 12).
Es imperativo que el Contador Público deba adquirir experticia tecnológica y lograr equilibrar sus competencias, de esta manera asegura su permanencia y éxito en este nuevo contexto. Además, los actores contables deben de establecer vínculos de acción profesional con expertos de otros campos del conocimiento que estén relacionados con la digitalización y sus procesos, como la ingeniería de software y sistemas. La educación formativa y continua tendrá que actualizar su malla curricular, modificando además sus perfiles de egreso y de esta manera brindar una formación acorde a las exigencias y necesidades del mundo 4.0. Solo así podrán asumir los retos y desafíos constantes y dinámicos que ya impuso la red y la automatización 4.0 de los procesos productivos, de circulación y distribución (Gómez, 2019).

El trabajo del auditor también se ha visto impactado con los cambios exponenciales de las TIC; ha pasado de las hojas de trabajo tabulares de columnas a las hojas de cálculo electrónicas, o incluso a formatos más sofisticados de algún ERP. Incluso la normativa fiscal verde o protectora del ambiente, premia con descuentos referenciados impositivos al uso de facturación electrónica. Es posible que en países en vías de desarrollo, el trabajo del auditor se realice digitalmente en un cien por ciento. Como lo expresa Hernández (2018) "esta situación permite al Contador Público tener otro tipo de enfoque, inclusive, diversificar sus servicios y buscar que los mismos sean acordes a los tiempos actuales" (párr. 13).

Los actuales cambios que se presentan en el área de servicios de auditores responden a la transformación digital 4.0 que va creciendo incesantemente. La auditoría, por consiguiente, debe preparase en este nuevo y exigente contexto y ofrecer un servicio actualizado basado en la tecnología 4.0, que incluye nuevas habilidades, potencia el juicio con experticia y competencia (De Pablos, 2016).

De igual modo, estos cambios tecnológicos afectan a la profesión de auditorías, en virtud de los nuevos requerimientos de las organizaciones empresariales y la alta competencia existente en la oferta de servicios. Es fundamental que la profesión de auditoría se sumerja y reinvente en este novísimo proceso de transformación. La proactividad debe ser una de las características de la auditoria de hoy, que le permita analizar las tendencias tecnológicas $4.0 \mathrm{y}$ a partir de ello, transformar el enfoque de servicios, maximizando su capacidad de asimilación de las nuevas oportunidades para incluir las nuevas tecnologías. Solo así una organización de auditoría estará vigente en el mercado y gozando de vigente credibilidad profesional (Instituto de Censores Jurados de Cuentas de España, 2019).

Finalmente, como reflexión concluyente, hay un largo camino en nuestro contexto en vía de desarrollo para responder 
a los desafíos y retos de la revolución 4.0. En primer lugar, el sistema universitario debe responsabilizarse por desarrollar las competencias genéricas del futuro contador auditor, innovando y actualizando sus mallas curriculares acorde a los nuevos cambios y requerimientos de las empresas en el mundo. La academia requiere de una acción planificada en el mediano y largo plazo, en consonancia a una política sectorial que asigne los recursos necesarios para la formación holística de un profesional de contaduría, competente y que demuestre capacidades, habilidades y destrezas para afrontar la revolución 4.0, sin dejar de lado lo más importante: la ética profesional y su actitud valorativa anticorrupción. En segundo lugar, los profesionales de la contabilidad y auditoría en ejercicio, quienes aún siguen con las prácticas tradicionales y que cada vez se sienten relegados a los sectores empresariales de mínima importancia, deben asumir conscientemente un cambio en su estructura de pensamiento y optar por la formación continua para conseguir una actualización digital que les permita ser incluido en la vorágine mundo 4.0 cada vez más competitivo (Araya, 2019).

\section{MATERIALES Y MÉTODOS}

El trabajo de investigación es de tipología cualitativa, documental no experimental, por cuanto no se ha manipulado las variables; sino que se ha observado el fenómeno a través del análisis, la crítica y la comparación de diversas fuentes de información, considerando autenticidad, credibilidad, representación y significado. El entorno contextual del estudio fue de nivel global, regional y pertinentemente en el ámbito nacional.

El proceso documental estuvo basado en la búsqueda, recuperación, análisis, crítica e interpretación de data secundaria, esto es, aquella que fue obtenida y registrada por diversos investigadores en fuentes documentales de modalidad impresa, audiovisual o electrónica. Asimismo, las fuentes correspondieron a bases de datos actualizados e indizados que publican artículos científicos arbitrados por pares ciegos en revistas científicas de ciencia económica, ciencias contables, ingeniería de sistemas e informática.

\section{RESULTADOS}

La cuarta revolución industrial o revolución digital 4.0 trajo consigo una vorágine de significativos cambios en la economía mundial y su réplica en Latinoamérica y en el Perú. El fenómeno de la digitalización se manifiesta en los procesos de producción, circulación y distribución de bienes y servicios a través de la red internacional, internet de las cosas y la inteligencia artificial.

La transformación en la industria 4.0 requiere de un sinnúmero de habilidades y destrezas para unir tecnologías y lograr la optimización de los procesos productivos. Se trata de una transformación inexcusable e imprescindible para toda organización que visione agilizar sus operaciones y lograr competitividad en un mercado con exigencias cada vez mayores, en el que las empresas y negocios están convocadas imperativamente a la mejora continua de sus procesos.

En la figura 1, se aprecia cuatro avances que se constituyen en impulsadores que favorecen la transformación digital industrial. La data recogida en el mundo físico se procesa, analiza y almacena digitalmente, convirtiéndose en información digital. Las labores manuales y repetitivas se sustituyen por sistemas autónomos, simplificados y automáticos. Las etapas de preproducción, producción y posproducción se sincronizan en un flujo integrado inteligente que permite productividad mayor, sostenibilidad y rentabilidad. Un ente importantísimo, el cliente, se presenta activamente informado y conectado con el producto y genera más oportunidades de negocio.

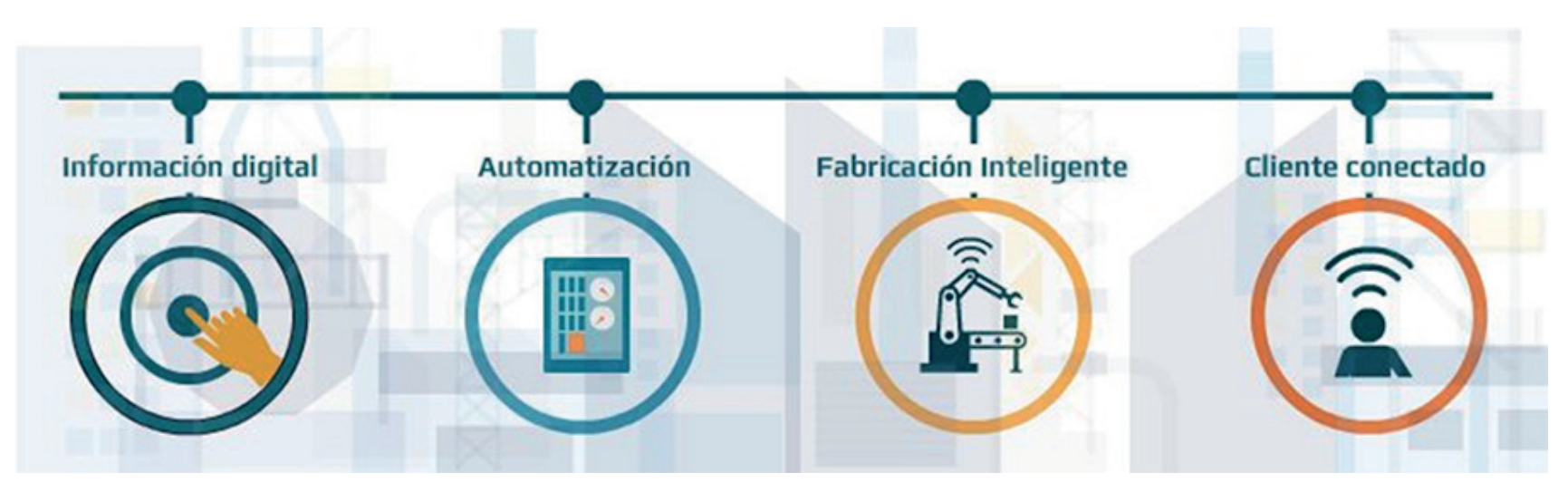

Figura 1. Impulsadores de la digitalización industrial Fuente: Grupo Garatu 
La revolución industrial 4.0 trastoca también el mercado laboral y el empleo. Al transformar el modo de producción y el comercio, sin duda influye significativamente en los vínculos entre trabajadores, empleados y profesionales con las empresas u organizaciones. Existen dos corrientes, la que asume que la cantidad de colaboradores se reducirán, y la otra, con los que mencionan que serán más. Sin duda hay empleos más fáciles de automatizar y tienden a ser reemplazados por robots o sistemas digitales; mientras los que no los son, deben desarrollar nuevas habilidades para adaptarse al mundo 4.0 competitivamente. Estas habilidades requeridas para mantenerse en los trabajos y labores del nuevo contexto habrán cambiado significativamente, respecto al actual.

En la tabla 1 se muestra, por un lado, una caída continua de la demanda de habilidades manuales y habilidades físicas y, por otro lado, una disminución en la demanda de habilidades relacionadas con la gestión de recursos financieros y con las habilidades de instalación y mantenimiento de tecnología. Las habilidades que continúan creciendo en importancia hacia el 2022 incluyen el pensamiento analítico e innovación, así como el aprendizaje activo y las estrategias de aprendizaje y las habilidades humanas como creatividad, originalidad e iniciativa. Aparece como una habilidad de importancia, el diseño y programación de tecnología. Tienden a caer, el pensamiento crítico, el análisis y la resolución de problemas complejos.

La ciencia contable no es ajena a los cambios de la globalización y la internacionalización digital, como profesión debe expandir sus horizontes hacia la internacionalización de los mercados financieros, los adelantos de las TIC y la Big Data. Sin embargo, la realidad muestra que los profesionales de contaduría y auditoría no están preparados para asumir los desafíos de la cuarta revolución. Por consiguiente, el experto contable y auditor debe adaptarse y reconvertirse, empezando con un cambio de chip e iniciar con el desarrollo de habilidades y competencias profesionales que le permita seguir vigente.

En la figura 2, se muestra el nivel de satisfacción de la formación recibida en materia digital en facultades de Ciencias Contables peruanas, un $74 \%$ de estudiantes manifiesta una valoración poco satisfactoria y apenas un $5 \%$, muy satisfactoria.

En cuanto a la formación del profesional de contaduría, el sistema universitario es el responsable del desarrollo de las nuevas competencias en el novel contador-auditor. Ello exige la innovación y actualización de las mallas curriculares que respondan a la cuarta revolución, sin dejar de lado la responsabilidad social y ética anticorrupción.

Estos resultados tienen relación con lo que a continuación muestra la tabla 2, respecto de la competencia digital en auditoria de los Contadores Públicos en Latinoamérica.

En la tabla 2, se evidencia las carencias en cuanto al nivel tecnológico que presentan los contadores públicos en Latinoamérica. Más del $50 \%$ de profesionales entrevistados responden no mostrar suficiencia o experticia en

Tabla 1

Comparación de las 10 habilidades de mayor demanda para afrontar la revolución 4.0

\begin{tabular}{|c|c|c|}
\hline EN EL 2018 & TENDENCIA AL 2022 & EN DECLIVE AL 2022 \\
\hline Pensamiento analítico e innovación & Pensamiento analítico e innovación & Destreza manual, resistencia y precisión \\
\hline Resolución de problemas complejos & Aprendizaje activo y estrategias de aprendizaje & $\begin{array}{l}\text { Memoria, habilidades verbales, auditivas y } \\
\text { espaciales }\end{array}$ \\
\hline Pensamiento crítico y análisis & Creatividad, originalidad e iniciativa & Gestión de recursos financieros y materiales \\
\hline $\begin{array}{l}\text { Aprendizaje activo y estrategias de } \\
\text { aprendizaje }\end{array}$ & Diseño y programación de tecnología & Instalación y mantenimiento de tecnología \\
\hline Creatividad, originalidad e iniciativa & Pensamiento crítico y análisis & $\begin{array}{l}\text { Lectura, escritura, matemática y escucha } \\
\text { activa }\end{array}$ \\
\hline Atención al detalle, confiabilidad & Resolución de problemas complejos & Gestión de personal \\
\hline Inteligencia emocional & Liderazgo e influencia social & Control de calidad y conciencia de seguridad \\
\hline $\begin{array}{l}\text { Razonamiento, resolución de problemas } \\
\text { e ideación }\end{array}$ & Inteligencia emocional & Coordinación y gestión del tiempo \\
\hline Liderazgo e influencia social & $\begin{array}{l}\text { Razonamiento, resolución de problemas e } \\
\text { ideación }\end{array}$ & Habilidades visuales, auditivas y del habla \\
\hline Coordinación y gestión del tiempo & Análisis y evaluación de sistemas & Uso de tecnología, monitoreo y control \\
\hline
\end{tabular}

Fuente: Encuesta sobre el Futuro del Empleo 2018, Foro Económico Mundial. 


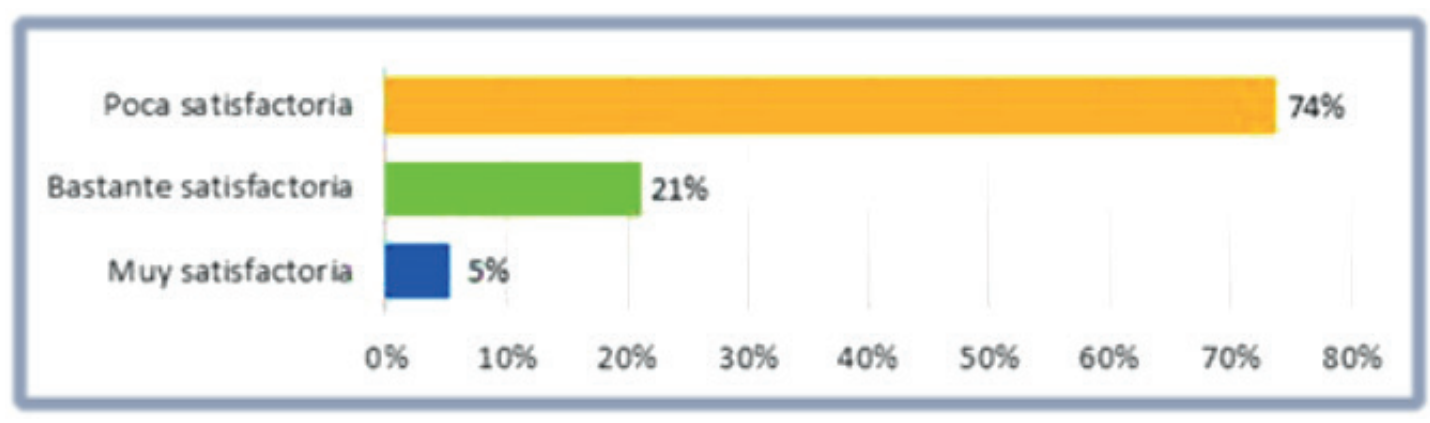

Figura 2. Valoración de la formación recibida en materia digital.

Fuente: Información obtenida de Díaz y León (2019)

Tabla 2

Nivel tecnológico de los contadores / auditores en Latinoamérica.

\begin{tabular}{|c|c|c|c|c|c|c|c|c|}
\hline \multirow{2}{*}{ Criterios } & \multicolumn{2}{|c|}{ sí } & \multicolumn{2}{|c|}{ NO } & \multicolumn{2}{|c|}{ NO RESPONDE } & \multicolumn{2}{|c|}{ TOTAL } \\
\hline & $\mathrm{n}$ & $\%$ & $\mathrm{n}$ & $\%$ & $\mathrm{n}$ & $\%$ & $\mathrm{~N}$ & $\%$ \\
\hline Conoce técnicas automatizadas de auditoría & 50 & 25 & 146 & 73 & 4 & 2 & 200 & 100 \\
\hline Utiliza técnicas automatizadas de auditoría & 60 & 30 & 130 & 65 & 10 & 5 & 200 & 100 \\
\hline $\begin{array}{l}\text { Conocimiento del flujo de la información por medio de los sistemas } \\
\text { de información (ERP) }\end{array}$ & 80 & 40 & 108 & 54 & 12 & 6 & 200 & 100 \\
\hline Uso experto de software contable e impositivo & 44 & 22 & 152 & 76 & 4 & 2 & 200 & 100 \\
\hline Utiliza software para la auditoría & 30 & 15 & 162 & 81 & 8 & 4 & 200 & 100 \\
\hline Está capacitado en reconocimiento de evidencia digital & 28 & 18 & 152 & 76 & 12 & 6 & 200 & 100 \\
\hline Usa muestreo aleatorio en trabajo de auditoría & 80 & 40 & 104 & 52 & 16 & 8 & 200 & 100 \\
\hline Manejo de plataformas online y espacios de co-working & 40 & 20 & 140 & 70 & 20 & 10 & 200 & 100 \\
\hline Aplica pruebas digitales de auditoría & 56 & 28 & 132 & 66 & 12 & 6 & 200 & 100 \\
\hline $\begin{array}{l}\text { Conocimientos de bases de datos estructuradas y no estructuradas } \\
\text { para extraer, transformar y analizar grandes volúmenes de infor- } \\
\text { mación }\end{array}$ & 70 & 35 & 114 & 57 & 16 & 8 & 200 & 100 \\
\hline
\end{tabular}

Fuente: En base a la información de encuesta realizada por Thomson Reuters (2020).

cuanto a sus habilidades digitales en el desempeño de la auditoría contable.

\section{DISCUSIÓN}

El estudio busca evidenciar, por un lado, si los Contadores Públicos en el Perú están capacitados para enfrentar la cuarta revolución industrial. La respuesta es contundente: no están en la capacidad para desenvolverse en el nuevo contexto 4.0 digital y vorágine. Por otro lado, persigue el análisis de las competencias profesionales de la contaduría para prestar servicios en los entes económicos digitalizados. Nuevamente la respuesta es categórica: las competencias que demuestran los profesionales de contabilidad no les sirven para actuar en empresas digitalizadas. Carpio,
Cruz y Martínez (2019) concluyen de modo similar al afirmar que los contadores y auditores salvadoreños no tienen las competencias para actuar en el contexto de la cuarta revolución. Esto representa un desafío para adquirir nuevos conocimientos, aptitudes y habilidades que garanticen su supervivencia en el ámbito laboral de la era tecnológica. En el mismo sentido, Grisanti (2017) concluye que el impacto de las TIC para el contador público ha sido contundente $\mathrm{y}$, por consiguiente, la revolución 4.0 exige al profesional una adaptación y reconversión en su formación y desempeño. En cuanto al proceso formativo y de actualización del profesional de contaduría, se debe considerar cambios sustantivos a nivel curricular acorde a la nueva economía 4.0; tal como sostiene Changmarín (2019), el contador público no será desplazado por los 
sistemas de información digitales mientras sea sujeto de una capacitación holística, actualizada y de acuerdo con los nuevos cambios tecnológicos. Esa tarea debe ser asumida por los colegios profesionales y las universidades. En síntesis, se concluye que la revolución 4.0 es dinámicamente ineludible, pero a la vez, permite la reflexión y búsqueda de oportunidades para que los profesionales de la contabilidad y auditoría enfrenten los retos y desafíos reinventándose y adquiriendo nuevas habilidades blandas y competencias digitales que le permitan no solo evitar su desplazamiento en el mercado laboral profesional, sino el éxito y crecimiento en esta nueva economía. La cuarta revolución industrial o revolución 4.0 trajo consigo para los Contadores Públicos un reto que se debe cumplir inexorablemente, ya que la innovación digital dejó de ser optativa, se ha convertido en una obligación, pero de ninguna manera es una amenaza, sino un verdadero acicate para el desarrollo de tan importante profesión.

\section{REFERENCIAS}

Amésquita, P. (2018). La cuarta revolución industrial y algunas implicaciones en las escuelas de negocios. $\mathrm{Pa}$ lermo Business Review, (18), 185-200.

Araya, S. (2019). Competencias genéricas de los estudiantes de Auditoría requeridas por las Big Four en Chile. Cuadernos de Contabilidad, 20(49). doi.org/10.11144/ Javeriana.cc20-49.cgea

Basco, A., Beliz, G., Coatz, D. y Garnero, P. (2018). Industria 4.0: fabricando el futuro. Recuperado de: https://publications.iadb.org/en/industria-40-fabricando-el-futuro

Blanco, F., Castro, J., Gayoso, R. y Santana, W. (2019). Las claves de la Cuarta Revolución Industrial. Cómo afectará a los negocios y a las personas. Barcelona: Libros de cabecera S.L.

Carpio, E., Cruz, V. y Martínez, C. (2019). Fortalecimiento de las competencias del profesional en contaduría para ejercer como auditor interno con base en los nuevos requerimientos de la cuarta revolución industrial 4.0 (Tesis de licenciatura). Universidad de El Salvador.

Ceballos, C. (9 de julio de 2018). 7 consejos para los Contadores Públicos vs la Inteligencia Artificial. Recuperado del sitio web: https://contadormx. com/2018/07/09/7-consejos-para-los-contadores-publicos-vs-la-inteligencia-artificial/

Changmarín, C. (2019). La Formación integral del contador público panameño con crecientes regulaciones en una economía digital y de la Big Data. Contabilidad y Auditoría, (50), 109-140.
De Pablos, E. (2016). Revolución Industrial 4.0 - Cambiará no solo lo que hacemos, sino también lo que somos. CyC Prisma, (21), 10-25. Recuperado de: http://media. investis.com/C/Catalana-occidente/documents/prisma21-comleto-baja.pdf

Deloitte Insights. (2018). The Fourth Industrial Revolution is here-are you ready? Recuperado de: https://www2. deloitte.com/content/dam/insights/us/articles/4364_ Industry4-0_Are-you-ready/4364_Industry4-0_Areyou-ready_Report.pdf

Echeverría, B. y Martínez, P. (2018). Revolución 4.0, competencias, educación y orientación. Revista Digital de Investigación en Docencia Universitaria, 12(2), 4-34.

Florez, M. (2012). Era digital, la nueva realidad del Contador Público. Adversia, (1). Recuperado de: https:// revistas.udea.edu.co/index.php/adversia/article/ view/11299/10332

Gómez, M. (2019). Pensando en el futuro: competencias profesionales del contador público frente a la cuarta revolución industrial. Recuperado de: https://docs.google. com/viewerng/viewer?url=http://media.actualicese. com/Pensando-en-el-futuro-Mauricio-Gomez-Villegas.pdf

Grisanti, A. (2017). Perspectivas de la contaduría pública en los tiempos de la era digital. Revista Visión Contable, 16, 96-119. doi:10.24142/rvc.n16a5

Hernández, E. (15 de octubre de 2018) Auditoría electrónica, tecnología al servicio del Contador Público. Recuperado de: http://contaduriapublica.org.mx/2018/10/15/auditoria-electronica-tecnologia-al-servicio-del-contador-publico/

Instituto de Censores Jurados de Cuentas de España. (2019). Transformación digital en el sector de auditoría. Madrid: Auditores.

Mantilla, S. (22 de abril de 2019). La contaduría digital funciona sin los contadores tradicionales ¿Tomó usted nota? [Mensaje en blog]. Recuperado de: https://samantilla1.net/la-contaduria-digital-funciona-sin-los-contadores-tradicionales/

Mayor, J., Pacheco, D., Patiño, J. y Ramos, S. (2018). Análisis de la integración del Big Data en los programas de contaduría pública en universidades acreditadas en Colombia. Revista CEA, 5(9), 53-76. doi. org/10.22430/24223182.1256

Peña, P. (2018). La era digital y sus efectos en la Tributación. Auditoría \& Finanzas, 187, 8-9. 
Quijano, D. (1 de marzo de 2020). Innovación: Pasado, presente y futuro del contador público en Colombia y en el mundo. Recuperado de: https://www.bdo.com.co/ es-co/publicaciones/boletines-audit/innovacion-pasado,-presente-y-futuro-del-contador-publico-en-colombia-y-en-el-mundo
Schwab, K. (2016). La cuarta revolución industrial. Recuperado de: http://40.70.207.114/documentosV2/ La\%20cuarta\%20revolucion\%20industrial-Klaus\%20 Schwab\%20(1).pdf 
